\title{
Combined Immunotherapy against Cancer: Limited Efficacy of Transcutaneous Immunization and Low-dose Cyclophosphamide
}

\author{
Julia Umansky ${ }^{1}$, Michael Weber ${ }^{1}$, Hansjörg Schild ${ }^{1}$, Markus P. Radsak ${ }^{2}$, Pamela Stein ${ }^{3, *}$ \\ ${ }^{1}$ Institute for Immunology, Johannes Gutenberg University Medical Center, Germany \\ ${ }^{2}$ IIIrd Department of Medicine, Johannes Gutenberg University Medical Center, Germany \\ ${ }^{3}$ Center for Thrombosis and Hemostasis, Johannes Gutenberg University Medical Center, Germany
}

Copyright (C) 2015 Horizon Research Publishing All rights reserved.

\begin{abstract}
Transcutaneous immunization (TCI) is a novel vaccination strategy with a promising potential for combating tumors or persistent infectious diseases. However, imiquimod-based TCI, we have previously developed, shows only limited effectiveness in terms of tumor protection, partly due to suppression by regulatory $\mathrm{T}$ (Treg) cells. To improve the vaccination potency we combined TCI with the cytotoxic drug cyclophosphamide (Cy) that is used for the treatment of tumors and described to mediate inactivation of Treg cells at low doses. Cy only slightly reduced Treg cell numbers in a concentration dependent manner under the chosen conditions, but also enhanced DC activation. Therefore, we used Cy-TCI in a therapeutic tumor assay where E.G7 lymphomas were subcutaneously transplanted and allowed to grow until palpable before the treatments started. Interestingly, the rates of tumor protection in TCI or Cy-TCI treated groups were identical. Towards the underlying mechanisms of the failure of $\mathrm{Cy}$-TCI to provide enhanced tumor protection, we observed increased numbers of monocytic and granulocytic immature myeloid cells after Cy-TCI, partly suppressing TCI-induced immune responses. Taken together, we suggest that $\mathrm{Cy}-\mathrm{TCI}$ induces inhibitory mechanisms counterregulating TCI enhancing effects, therefore suppressing vaccination-induced immune responses.
\end{abstract}

Keywords Transcutaneous Immunization, Cyclophosphamide, Regulatory T Cells, Immature Myeloid Derived Cells, Dendritic Cells

\section{Introduction}

Therapeutic cancer vaccines have to unify several characteristics to successfully combat solid tumors. Beside the generation of adequate $\mathrm{T}$ cell responses against tumor cells vaccinations should also target suppressive occurrences like immunosuppressive molecules, secreted by the tumor, or regulatory cells of the immune system, recruited or activated by the tumor [1-3]. In this context we have recently described a transcutaneous immunization (TCI) method, based on the synthetic Toll-like Receptor 7 agonist imiquimod [1-6], which is the active component of the commercially available Aldara crème. When applied to the skin, imiquimod induces inflammatory responses [7-10] and is also effective in patients with HPV-associated or malignant skin diseases [1-3]. TCI with imiquimod directly addresses the skin associated lymphoid tissue (SALT) to initiate adaptive immunity [4-6]. We and others have shown that a potent and specific primary $\mathrm{T}$ cell response is induced upon vaccination with Aldara and a cognate cytotoxic $\mathrm{T}$ lymphocyte (CTL) epitope [7-10]. In this situation increased numbers of bone-marrow derived $\mathrm{CD} 11 \mathrm{c}^{+}$dermal dendritic cells (DCs) migrate from the skin in the draining lymph nodes and function as the essential antigen-presenting cells (APCs) $[11,12]$. However, CTL responses induced by TCI with imiquimod and peptide rapidly fade away, resulting in poor memory formation and only partial tumor protection, shown by us for transplanted EG.7 tumors [13] and also by others in B16 melanoma models [6,14]. Towards the immunological limitations of this vaccination strategy, we have demonstrated that IL-10 and regulatory T cells (Treg) contribute to the suppression of TCI-induced immune responses $[2,15]$. Another relevant part of Treg-mediated suppression in our context may also be mediated via CTLA4 $[16,17]$, a major suppressive pathway of Treg cells and also relevant in tumor-induced suppressive environments [18].

The cytotoxic drug cyclophosphamide (Cy) is a nitrogen mustard-alkylating agent that is used for the treatment of various diseases. Until now, the molecular mechanisms of $\mathrm{Cy}$ are not fully understood, but there is a correlation between dose and biological activity [19]. Higher doses of $\mathrm{Cy}$ are applied during bone-marrow transplantations and in refractory rheumatoid diseases as immunomodulatory drug $[20,21]$. Beyond this, also low doses of $\mathrm{Cy}$ are effective in 
the treatment of melanoma [22,23] and autoimmune disorders [24]. On the cellular level, Cy can lead to enhanced immune responses by increasing for example the differentiation of sensitized CTL precursors into effective CTLs [25-27]. Furthermore, Cy can induce lymphodepletion, subsequently leading to expansion of immature DC in peripheral blood [28], as well as decreasing Treg numbers and also inhibit their suppressive capacities [29].

In this study we combined our TCI protocol with low-dose Cy treatment to prevent suppression by Treg cells and to induce a specific immune response and enhanced tumor protection. Low dose $\mathrm{Cy}$ did not diminish vaccination-induced immune responses and led to an enhanced DC activation concurrent with a reduction of Treg cells after vaccination. However, despite an enhanced DC activation and Treg cell depletion, Cy-TCI did not result in an enhanced CTL response or tumor protection compared to TCI alone. Hypothesizing that $\mathrm{Cy}$ induces additional inhibitory factors after combined treatment, we detected increased numbers of monocytic as well as granulocytic immature myeloid cells in the spleens of Cy-TCI treated mice along with a reduced CTL activation capacity ex vivo compared to TCI or untreated controls. Taken together, the combination of low-dose Cy with TCI does not diminish vaccination-induced immune responses. Therefore, such combination appears feasible, but we suggest that $\mathrm{Cy}$ also induces inhibitory mechanisms that suppress vaccination-induced immune responses and may impair the overall effectiveness of immunotherapeutic treatments.

\section{Material and Methods}

\subsection{Mice}

C57BL/6 mice at the age of 6-8 weeks were obtained from the local animal facility of the University of Mainz. DEREG mice on C57BL/6 background were kindly provided by $\mathrm{T}$. Sparwasser [30]. All animal work performed in this study was conducted to the national guidelines and was reviewed and confirmed by an institutional review board/ethics committee headed by the local animal welfare officer. The responsible national authority finally approved the animal experiments, which is the National Investigation Office Rheinland-Pfalz (Koblenz, Germany). The Approval ID assigned by this authority: AZ 23 177-07/G08-1-023 and G13-1-012.

\subsection{Transcutaneous Immunizations}

Transcutaneous immunizations with Imiquimod were described previously [10]. Briefly, dorsal hair was removed using electric clippers. $100 \mu \mathrm{g}$ SIINFEKL-Peptid $\left(\mathrm{OVA}_{257-264}\right.$, kindly provided by Dr. Stevanovic, Department Immunology, Institute for Cell Biology, University of Tübingen, Germany) was added to $50 \mathrm{mg}$ crème containing $5 \%$ Imiquimod (Aldara, Meda) and applied on the dorsal skin of anesthetized mice on days 0 and 1 .

\subsection{Cyclophosphamide Treatment}

Mice were injected i.p. with $25 \mathrm{mg} / \mathrm{kg}, 50 \mathrm{mg} / \mathrm{kg}$ or $100 \mathrm{mg} / \mathrm{kg}$ cyclophosphamide one day prior to immunizations.

\subsection{Depletion of Regulatory $\mathrm{T}$ Cells}

Where indicated C57BL/6 mice were depleted of regulatory $\mathrm{T}$ cells by i.p. injection of an anti-CD25 Ab (PC61, $400 \mu \mathrm{g}$, day -4). For regulatory $\mathrm{T}$ cell depletion in DEREG mice received i.p. injections of diphtheria toxin $(1 \mu \mathrm{g})$ at days $-1,1$ and 3 .

\subsection{Tumor Rejection Assay}

Tumors were implanted by s.c. injection of $4 \times 10^{5}$ EG.7 thymoma cells (from ATCC) as described previously [13]. Treatments started when tumors get palpable (10-12 days after inoculation). Tumor size was monitored three times per week with a caliper measuring the diameter in two dimensions. Mice were killed when tumor size exceeded $20 \mathrm{~mm}$ in both diameters.

\subsection{Flow Cytometric Analyses and in vivo Cytotoxicity Assay}

The following mAbs were used for analyses by flow cytometry: -conjugated CD8 (clone 53-6.7), -conjugated CD44 (clone IM7), -conjugated CD62L (clone MEL-14), -conjugated CD4 (clone), -conjugated CD25 (clone 7D4), APC-conjugated FoxP3 (clone) all from BioLegend or eBiosciences. Blood samples were collected after tail vein incision and incubated on ice with specific mAbs as indicated after a hypotonic lysis step. For detection of SIINFEKL-specific T cells samples were stained with $\mathrm{H} 2-\mathrm{K}^{\mathrm{b}}$ tetramer. $\mathrm{H} 2-\mathrm{K}^{\mathrm{b}}-\mathrm{SIINFEKL}^{+}$cells were determined among gated $\mathrm{CD} 8^{+} \mathrm{T}$ cells. In vivo cytolytic activity was determined by transfer of carboxyfluorescein diacetate succinimidyl ester $\left(0.4 \mu \mathrm{M} \quad \mathrm{CFSE}^{\text {low }}\right.$ and $\left.4 \mu \mathrm{M} \quad \mathrm{CFSE}^{\text {high }}\right)$ labelled splenocytes $\left(2 \times 10^{7}\right.$ per mouse). The $\mathrm{CFSE}^{\text {low }}$ population was additionally loaded with SIINFEKL-peptide. Splenocytes of immunized and control mice were analysed by flow cytometry. All analyses were performed with a LSRII Flow Cytometer and FACSDiva software (BD Pharmingen, Hamburg, Germany).

\subsection{Preparation and Staining of Lymph Nodes}

C57BL/6 mice were treated as indicated. Inguinal, axillar and brachial lymph nodes were collected, picked and digested for 40 minutes with type 2 collagenase $(1 \mathrm{mg} / \mathrm{ml}$ from Worthington Biochemicals, Lakewood, NJ) and DNase I ( $100 \mu \mathrm{g} / \mathrm{ml}$, Sigma-Aldrich). The reaction was stopped by adding $10 \mathrm{mM}$ EDTA (Sigma-Aldrich). The cells were 
labelled with the following mAbs: APC-Cy7-conjugated CD11c (clone N418), Pacific Blue-conjugated MHC II (clone M5/114.15.2), -conjugated CD8 (clone 53-6.7), -conjugated CD4 (clone RM4-5), -conjugated CD80 (clone 16-10A1), -conjugated CD86 (clone GL-1) all from BioLegend or eBiosciences. Absolute cell numbers were calculated using Flow-Count Fluorospheres from Beckmann-Coulter according to the manufacturer's instructions.

\subsection{In Vitro Proliferation Assay}

C57BL/6 mice were treated as indicated over a period of three weeks. Spleens of experimental groups were pooled and single-cell suspensions were prepared. After a hypotonic lysis step cells were divided to three parts: i) $\mathrm{CD} 25^{+}$cells were depleted using CD25-specific magnetic beads and MACS sorting, further working with the flow through, ii) Ly6G ${ }^{+}$cells were depleted using Ly6G-specific magnetic beads and MACS sorting, further working with the flow through and iii) cells without any depletion (magnetic beads from Miltenyi Biotech, Bergisch-Gladbach, Germany). After incubation of cells with SIINFEKL $(10 \mathrm{ng} / \mathrm{ml})$ for 1 hour, cells were washed twice. Stimulator $\left(3 \times 10^{5}\right.$ cells/well $)$ and responder OT-1 splenocytes $\left(1 \times 10^{5}\right.$ cells/well $)$ were cocultured for three days, pulsed with ${ }^{3} \mathrm{H}$-thymidine $(0.5$ $\mu \mathrm{Ci} / \mathrm{ml}$; Perkin Elmer, Rodgau, Germany) and harvested the following day. ${ }^{3} \mathrm{H}$-thymidine incorporation was assessed with a 1205 betaplate reader (LKB Wallac, Turku, Finland).

\subsection{Statistical Analysis}

All statistical analyses were performed using GraphPad Prism (version 5.0a for Mac OS X, GraphPad Software, San Diego California USA, www.graphpad.com). Survival analyses were performed by the Mantel-Cox test. For comparison between two groups a two-tailed Student's $t$ test was used. Comparisons of multiple groups were performed by one-way ANOVA with Bonferroni's posttest. For all analyses, $\mathrm{p}<0.05$ was considered as statistically significant.

\section{Results}

\subsection{Low-dose Cyclophosphamide does not Impair Transcutaneous Immunization with Imiquimod}

We have previously shown that TCI with imiquimod elicits a potent primary CTL-response, but is diminished by the presence of Treg cells as well as IL-10 [2]. The prevention of Treg-mediated suppression either by reducing Treg-cell numbers or by inhibiting Treg-cell function might be a promising approach to enhance TCI-induced immune responses. Cy treatment is described to impede Treg-mediated suppression [29] and might therefore represent an interesting way to enhance TCI efficacy. To this end, we investigated the effects of various $\mathrm{Cy}$ amounts on the TCI-induced CTL response using the TLR7 ligand imiquimod together with the major histocompatibility complex class I-restricted T-cell epitope $\mathrm{OVA}_{257-264}$. One week after vaccination we determined the in vivo cytolytic activity of the induced CTLs. As depicted in Figure 1A, systemic administration of higher doses $(50 \mathrm{mg} / \mathrm{kg}$ and $100 \mathrm{mg} / \mathrm{kg}$ ) of Cy slightly decreased lysis of peptide-loaded target cells, whereas the lowest amount of Cy $(25 \mathrm{mg} / \mathrm{kg})$ had no impact on cytotoxicity compared to TCI alone. Additionally we assessed the frequency of peptide-specific CD8 T cells (Figure 1B). Again, higher doses (50 mg/kg and $100 \mathrm{mg} / \mathrm{kg}$ ) reduced induction of peptide-specific CD8 T cell responses compared to TCI alone. Nevertheless, the lowest dose of Cy $(25 \mathrm{mg} / \mathrm{kg})$ had no impact on CTL response indicated by equivalent amounts of peptide-specific $\mathrm{T}$ cells compared to TCI alone. Analysing the activation status of induced $\mathrm{CD} 8^{+} \mathrm{T}$ cells for low-dose $\mathrm{Cy}$ and $\mathrm{TCI}$ compared to TCI alone (Figure 1C) revealed no difference of CD44 and CD62L expression in both groups, leading to the conclusion that the additional application of low-dose Cy $(25 \mathrm{mg} / \mathrm{kg})$ does not diminish TCI induced priming of CTL-responses.

As the combination of low-dose Cy and TCI seemed to be feasible, we evaluated this vaccination protocol in terms of tumor protection (Figure 1D). We inoculated E.G7 thymoma cells, expressing chicken ovalbumin as a surrogate tumor antigen. After tumors became palpable (days 10-12), the mice were treated with TCI with or without injection of $\mathrm{Cy}$ $(25 \mathrm{mg} / \mathrm{kg})$. Cy treatment alone already enhanced survival of tumor-bearing mice with a median survival of 49 days compared to the untreated group (median survival 32.5 days), which was not significant compared to the untreated group ( $\mathrm{p}=0.15$ by Mantel Cox test). TCI alone and Cy-TCI showed identical results (median survival not reached), indicating that $\mathrm{Cy}$ neither reduces nor enhances survival compared to TCI alone, also in line with the former experiments concerning induction of CTL-responses. 
A
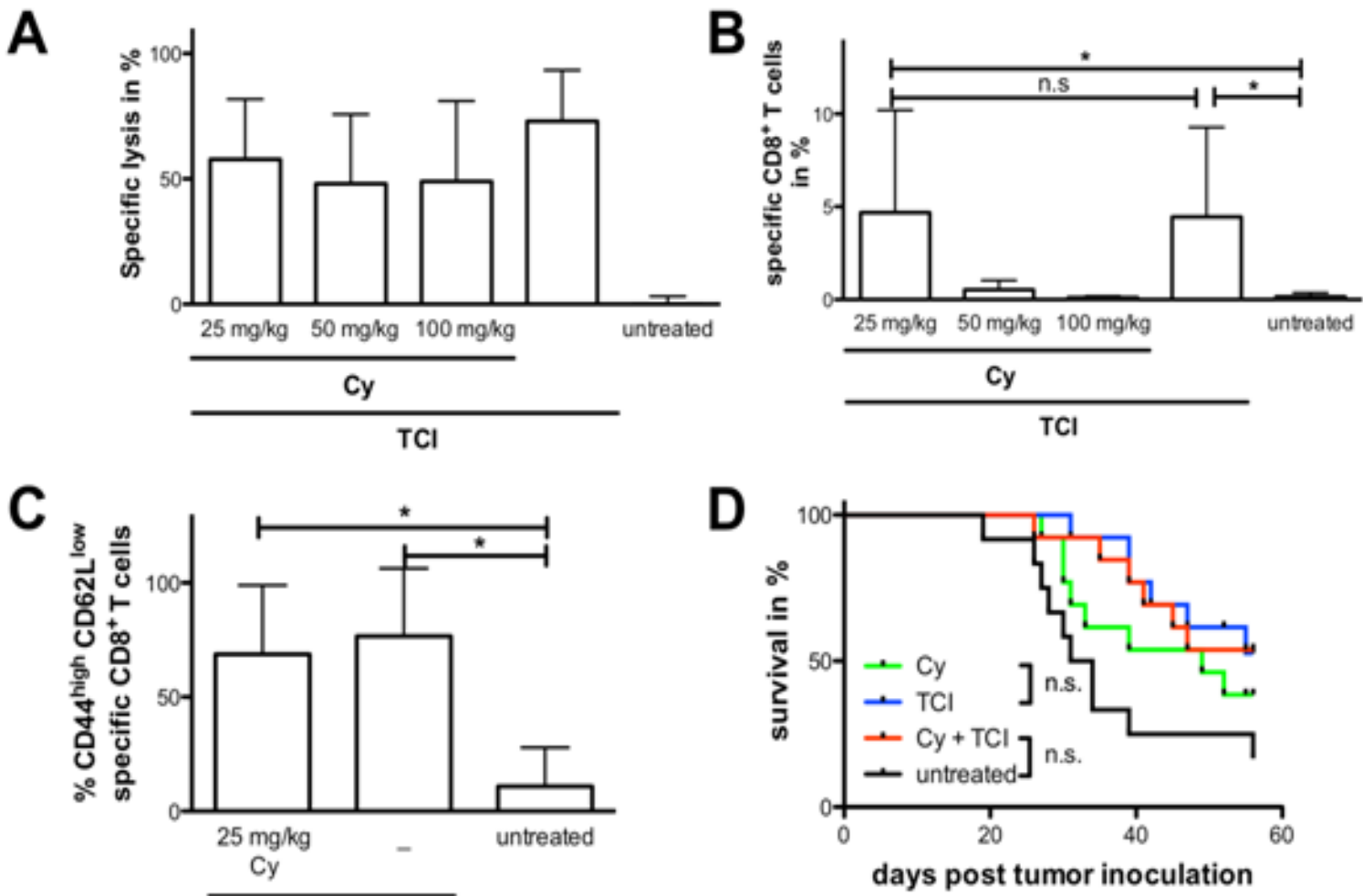

Figure 1. Low-dose cyclophosphamide does neither impair TCI-induced CTL-responses nor enhance survival in a therapeutic tumor model.

C57BL/6 mice were injected i.p. with various amounts of Cy as indicated (day -1). Transcutaneous immunizations were performed using Imiquimod-containing Aldara $5 \%$ crème $\left(50 \mathrm{mg}\right.$ ) together with $\mathrm{OVA}_{257-264}$ (SIINFEKL, $100 \mu \mathrm{g}$ ) at days 0 and 1 . A) Cytolytic activity was determined after adoptive transfer of peptide-loaded splenocytes. B) The frequency and C) activation phenotype of peptide-specific CD ${ }^{+}$T cells in the blood was assessed at day 6 using surface staining and flow cytometry. D) E.G7 cells $\left(4 \times 10^{5}\right)$ were injected subcutaneously into the flank of mice. Immunization treatments and Cy injection $(25 \mathrm{mg} / \mathrm{kg}$ ) started after 10-12 days when tumours became palpable. A-C) A cumulative analysis (mean with SD) of two independent experiments for $50 \mathrm{mg} / \mathrm{kg} \mathrm{Cy}$ or $100 \mathrm{mg} / \mathrm{kg}$ Cy (each $\mathrm{n}=6$ ) and five independent experiments with $\mathrm{n}=22$ for $25 \mathrm{mg} / \mathrm{kg} \mathrm{Cy}, \mathrm{n}=21$ for TCI and $\mathrm{n}=14$ for control is shown. D) A cumulative analysis of two independent experiments with $n=13$ for Cy-TCI, TCI and Cy alone and $n=12$ for untreated is shown. $(*)$ Significant difference $(p<0.05)$ by Mann-Whitney test, n.s not significant.
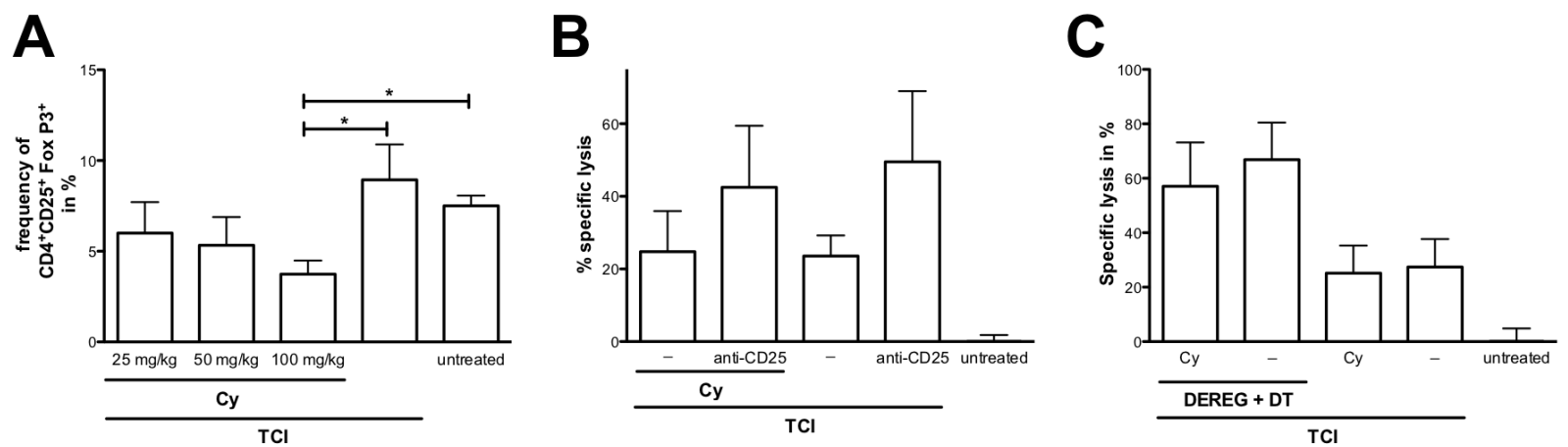

Figure 2. Low-dose cyclophosphamide does not completely deplete Tregs and has no major impact on their functionality

Mice were injected i.p. with Cy as indicated (day -1). At days 0 and 1 transcutaneous immunizations were performed. A) Groups received titrated amounts of $($ Cy $25 \mathrm{mg} / \mathrm{kg}, 50 \mathrm{mg} / \mathrm{kg}, 100 \mathrm{mg} / \mathrm{kg}$ ). Amounts of Treg cells in the spleens were analysed. B) Mice were treated with Cy $(25 \mathrm{mg} / \mathrm{kg})$ and TCI or TCI alone with or without the additional injection of an anti-CD25 Ab (clone PC61, $400 \mu \mathrm{g}$ i.p., day -4). C) FoxP3-diphteria toxin receptor transgenic (DEREG) mice were immunized with Cy $(25 \mathrm{mg} / \mathrm{kg}$ ) and TCI or TCI alone with or without depletion of Treg cells by application of diphtheria toxin (DT, $1 \mu \mathrm{g}$ i.p., days -1, $1,3)$. B,C) In vivo cytolytic activity was assessed after adoptive transfer of peptide loaded and carboxyfluorescein diacetate succinimidyl ester-labelled target cells. A cumulative analysis of two or three independent experiments is shown. $\left({ }^{*}\right)$ Significant difference $(\mathrm{p}<0.05)$ by Student's $t$ test.

\subsection{Low-dose Cy Depletes Treg Cells after TCI}

One major reason for combining $\mathrm{Cy}$ and TCI was its ability to inhibit the suppressive capacity of Treg cells or even deplete them [29]. As the treatment with low-dose Cy in our vaccination protocol did not increase the induction of a specific immune response, we repeated the titration of $\mathrm{Cy}$, immunized mice with TCI and stained for Treg cells in the spleens at day 6 when analysing the CTL-response. The amount of Treg cells (determined as $\mathrm{CD} 4^{+} \mathrm{CD} 25^{+} \mathrm{FoxP} 3^{+}$ cells) was slightly decreased after the application of $25 \mathrm{mg} / \mathrm{kg}$ or $50 \mathrm{mg} / \mathrm{kg}$ compared to the untreated control. This effect was more pronounced after treatment with $100 \mathrm{mg} / \mathrm{kg} \mathrm{Cy}$. In contrast, TCI alone led to a marginal non-significant increase in Treg cell numbers (Figure 2스). 
To determine the remaining suppressive activity of Treg cells after Cy-TCI, we additionally used an anti-CD25 monoclonal antibody (clone PC61, $400 \mu \mathrm{g}$, day -4) to deplete Treg cells before immunizations started. Additional depletion of $\mathrm{CD}^{2} 5^{+}$cells led to an enhanced immune response shown by increased lysis of peptide-loaded target cells by cytotoxic $\mathrm{T}$ cells, independent of additional low-dose Cy application (Figure 2B $)$. Since antibody-mediated depletion may exert secondary effects, we also employed another approach for Treg depletion by immunizing FoxP3-diphtheria toxin receptor transgenic mice (DEREG) with Cy-TCI or TCI alone. By administration of diphtheria toxin (DT) $\mathrm{FoxP}^{+}$cells are specifically ablated. Comparable to our published results [2] and in line with the former experiments using a monoclonal antibody, the absence of Treg cells promoted an increased CTL response compared to TCI without prior depletion. The additional application of $\mathrm{Cy}$ again had no impact on the detected immune response (Figure 2ㄷ). Taken together, these results implicate that either low-dose $\mathrm{Cy}$ administration has no sufficient impact on Treg cell numbers and functionality or it induces other suppressive factors leading to CTL responses comparable to TCI alone.

\subsection{Enhanced DC activation Phenotype after Cy-TCI}

For TCI-induced CTL responses, bone-marrow derived $\mathrm{CD}_{11 \mathrm{c}^{+}}$dermal DC are essential for priming of peptide-specific $\mathrm{T}$ cells [12]. As $\mathrm{Cy}$ is described to mediate expansion of immature DC as a result of lymphodepletion [28], we next investigated the influence of systemic administration of $\mathrm{Cy}$ on the local activation of DCs in the skin. We treated mice with TCI in the presence or absence of $\mathrm{Cy}$ in the additional presence of FITC on the shaved backs to identify skin-derived DCs in the draining lymph nodes. Three days after immunization the draining lymph nodes were harvested and analysed by flow cytometry (gating strategy Figure 3A). Gating on FITC ${ }^{+}$DCs we were unable to detect differences in the number of DCs after Cy-TCI compared to TCI alone (data not shown). Interestingly, the phenotype of migrated skin-derived DCs revealed a significantly increased expression of the activation markers CD80 (Figure 3B) and CD86 (Figure 3C) of Cy-TCI-treated mice compared to TCI alone or to FITC treated, but otherwise unimmunized controls. These data clearly show that Cy leads to an enhanced activation status of skin-derived DC.

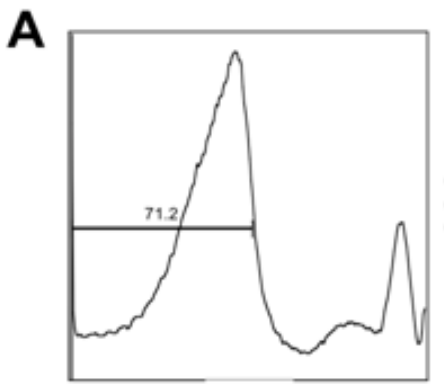

Pl

B

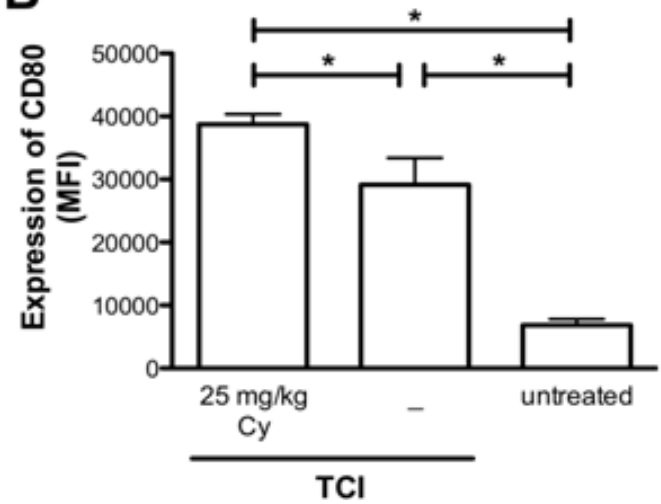

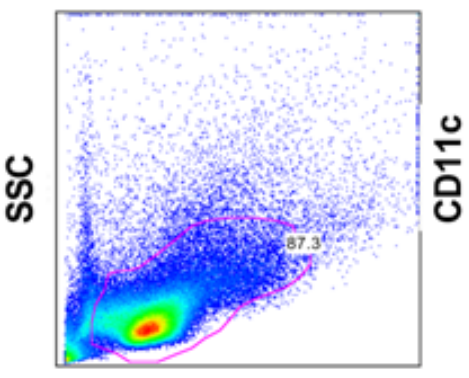

FSC

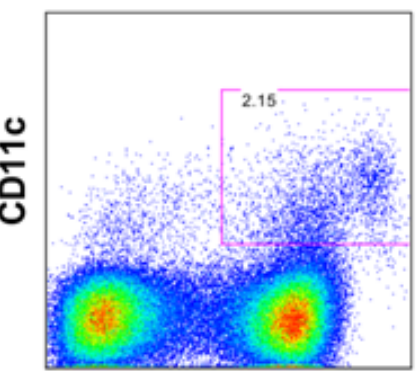

MHC class II

C

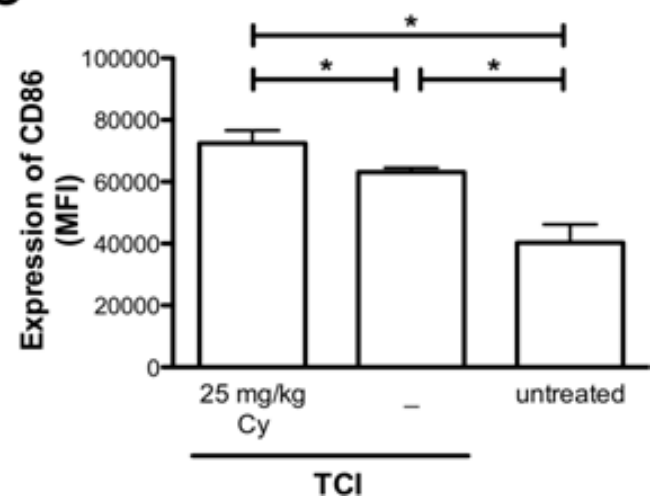

Figure 3 . Increased activation phenotype of skin-derived DC after cyclophosphamide application.

Mice were injected i.p. with Cy as indicated (day -1). At days 0 and 1 transcutaneous immunizations were performed. Additionally a FITC-solution was applied on the shaved back skin. Skin-draining lymph nodes we harvested at day 3, digested and analysed with flow cytometry. A) Skin-derived DC were determined as living cells with a typical FSC/SSC distribution as MHC class II CD1 1 $\mathrm{c}^{+}$and among these as FITC ${ }^{+}$. Mean fluorescence intensity of B) CD80 and C) CD86 were analysed. Depicted are representative results with $\mathrm{n}=4$ for 2 independent experiments. (*) Significant difference (p<0.05) by Students $t$ test. 
A

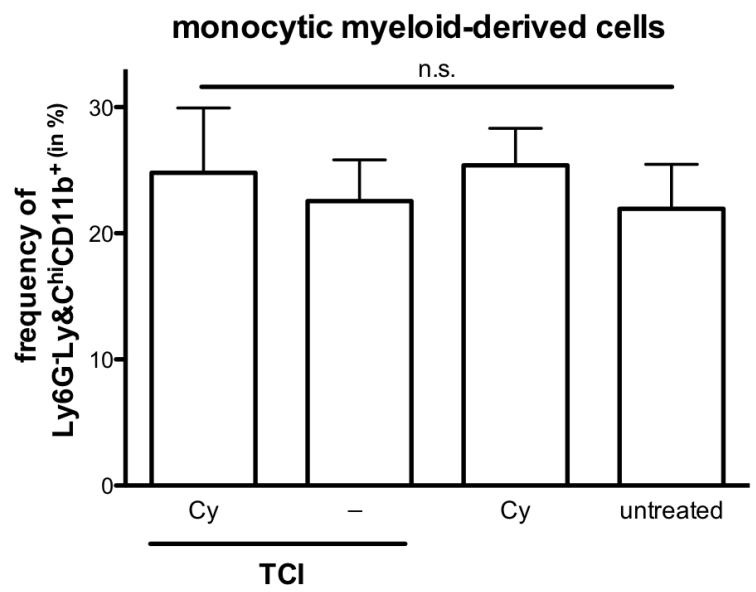

C

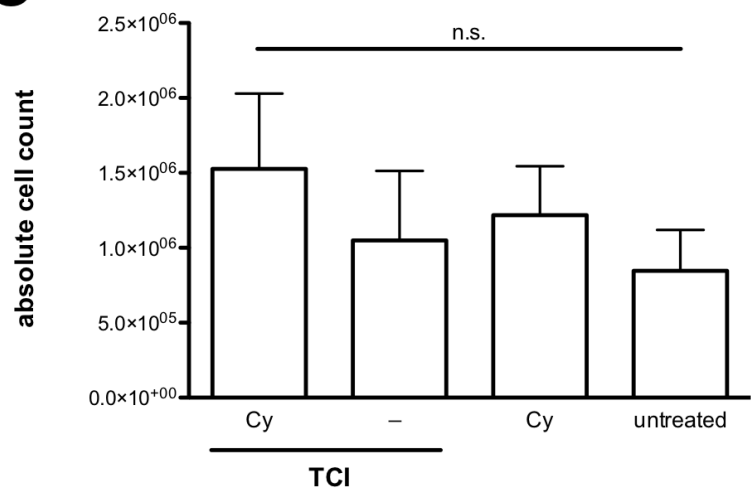

B granulocytic myeloid-derived cells

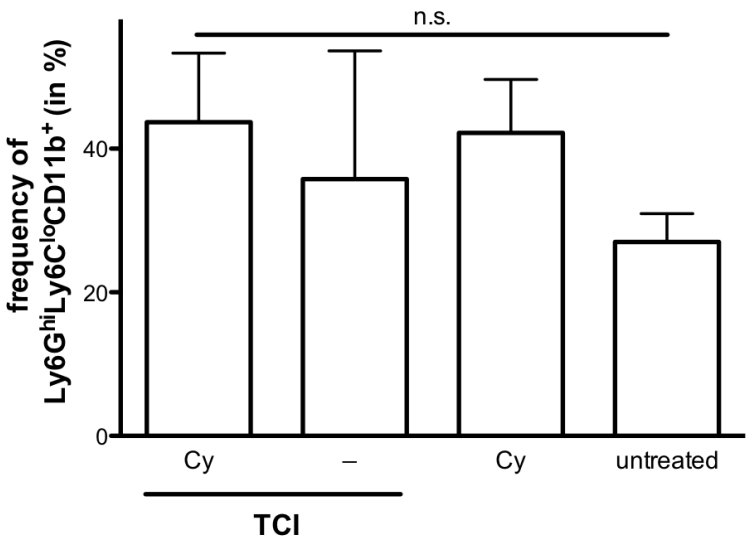

D

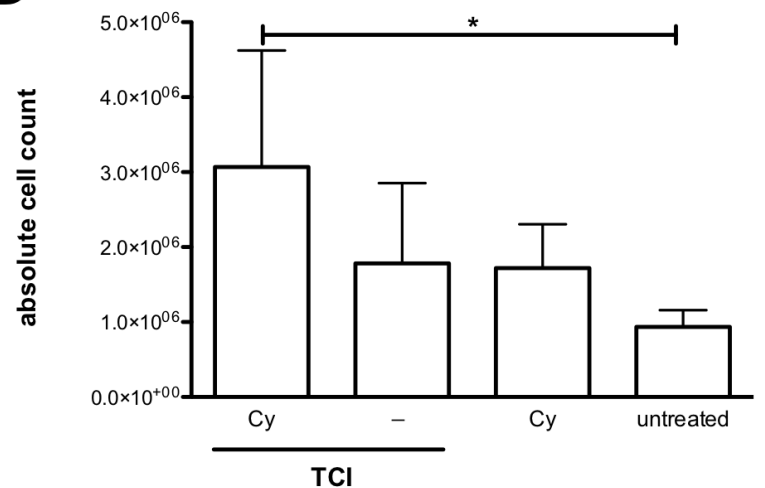

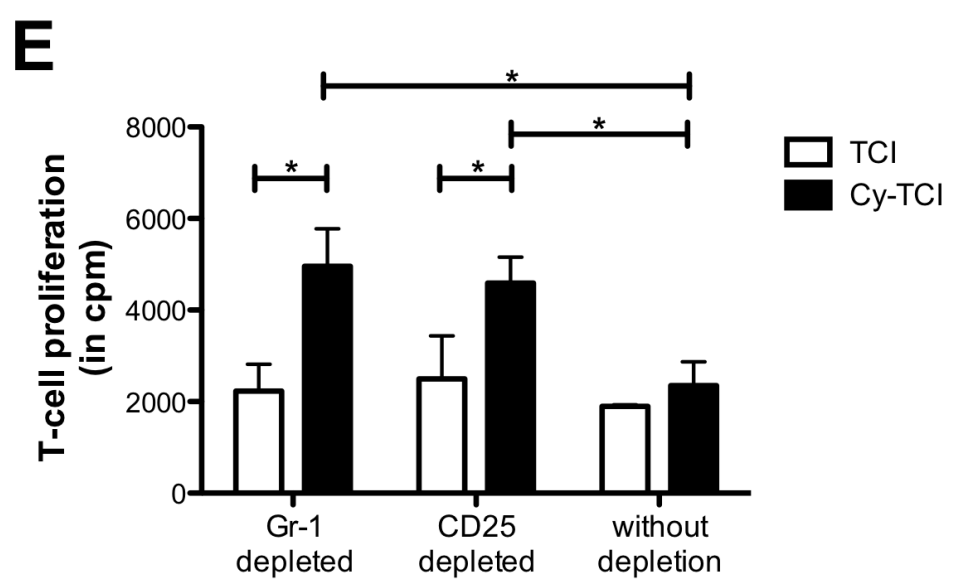

Figure 4. Monocytic as well as granulocytic immature myeloid cells are induced upon low-dose cyclophosphamide.

Mice were injected i.p. with Cy as indicated (day -1). At days 0 and 1 transcutaneous immunizations were performed. At day 6 samples from A,B) blood (relative amount gated on $\mathrm{CD}_{11 \mathrm{~b}^{+}}$) and $\left.\mathbf{C}, \mathbf{D}\right)$ spleen (absolute cell counts) were stained for monocytic (CD11b ${ }^{+}$Ly-6G ${ }^{-}$Ly6 $\mathrm{C}^{\text {hi }}$ ) and granulocytic $\left(\mathrm{CD} 11 \mathrm{~b}^{+} \mathrm{Ly}-6 \mathrm{G}^{+} \mathrm{Ly}-6 \mathrm{C}^{\mathrm{lo}}\right)$ immature myeloid cells and analysed by flow cytometry. Depicted are cumulative results of two independent experiments with $\mathrm{n}=8$ for Cy and TCI, TCI and Cy and $\mathrm{n}=4$ for control. (*) $\mathrm{p}<0.05$ by one-way ANOVA with Bonferroni's posttest n.s. = no significant difference E) Mice were treated with Cy-TCI or TCI alone over a period of three weeks. Afterwards spleens of immunized mice were depleted of Ly6G/C or CD25 ${ }^{+}$cells or left undepleted, loaded with SIINFEKL peptide and used as APCs for OT-1 T cells. Proliferation of T cells was assessed in a $\left[{ }^{3} \mathrm{H}\right]$ thymidine assay (in triplicate wells). (*) Significant difference $(\mathrm{p}<0.05)$ by Students $t$ test. 


\subsection{Cy-induced Immature Myeloid-derived Cells Suppress CTL Response Post TCI}

So far, we demonstrated that the CTL response induced by Cy-TCI was not diminished compared with TCI alone. However the depletion of Treg cells as well as the DC activation phenotype after Cy suggest that $\mathrm{Cy}$ should lead to an enhancement of the CTL response which was not the case. Therefore, we hypothesized that there might also be inhibitory factors induced by Cy treatment. Recently, Sevko and co-workers [31] demonstrated the induction of myeloid-derived suppressor cells (MDSCs) upon systemic injection of low-dose Cy. To elucidate whether such immature myeloid-derived cells were induced upon Cy-TCI, we treated mice with low-dose Cy $(25 \mathrm{mg} / \mathrm{kg})$ and TCI, TCI alone or $\mathrm{Cy}$ alone and stained for monocytic $\left(\mathrm{CD} 11 \mathrm{~b}^{+} \mathrm{Ly}-6 \mathrm{G}^{-} \mathrm{Ly} 6 \mathrm{C}^{\mathrm{hi}}\right)$ and granulocytic $\left(\mathrm{CD} 11 \mathrm{~b}^{+} \mathrm{Ly}-6 \mathrm{G}^{+} \mathrm{Ly}-6 \mathrm{C}^{\mathrm{lo}}\right)$ immature myeloid-derived cells [32] in blood and spleen, five days after treatment. As depicted in Figure 4A, no induction of monocytic immature myeloid-derived cells could be observed in the blood after $\mathrm{Cy}$ treatment, whereas some granulocytic immature myeloid-derived cells (Figure 4B) were detectable. Conversely, the absolute cell numbers in the spleens of treated mice revealed that TCI alone showed no induction of the monocytic subtype (Figure 4C), but a significant induction of granulocytic cells ( $p<0.05$ by one-way ANOVA with Bonferroni's posttest; Figure 4D). Cy alone showed a comparable amount of monocytic as well as granulocytic cells compared to TCI alone. These results indicate that $\mathrm{Cy}$ induces suppressive immature myeloid cells.

To further evaluate whether the induced monocytic and granulocytic immature myeloid-derived cells contribute to the suppression of TCI-induced CTL responses, we immunized mice over a period of three weeks in the presence or absence of Cy. On day 5 after the last treatment, we took the spleens of mice and either depleted $\mathrm{Ly} 6 \mathrm{G} / \mathrm{C}^{+}$cells (targeting immature myeloid cells), $\mathrm{CD} 25^{+}$cells (targeting Treg cells) or left the splenocytes undepleted. Afterwards we co-cultured these splenocytes with TCR-transgenic T cells from OT-1 mice, recognizing the $\mathrm{H} 2-\mathrm{K}^{\mathrm{b}}$-restricted epitope $\mathrm{OVA}_{257-264}$ to subsequently analyse the induced T-cell proliferation by $\left[{ }^{3} \mathrm{H}\right]$ thymidine incorporation assay. As depicted in Figure 4E, we detected a strong proliferation of OT-1 T cells using splenocytes from TCI-treated mice as APCs. Notably, this was independent of the cell-type depleted before co-culture. Undepleted splenocytes from Cy-TCI treated induced OT-1 T-cell proliferation in a comparable way to TCI alone. Interestingly, the depletion of either $\mathrm{Ly} 6 \mathrm{G} / \mathrm{C}^{+}$or $\mathrm{CD} 25^{+}$cells from Cy-TCI treated donor cells, significantly increased $\mathrm{T}$-cell proliferation further underlining the idea that $\mathrm{Cy}$ induces inhibitory factors that suppress TCI-induced immune responses.

All together, these data demonstrate that Cy treatment leads to the induction of monocytic and granulocytic immature myeloid-derived cells, that an addition to regulatory $\mathrm{T}$ cells contribute to the suppression of induced
CTL response

\section{Discussion}

Transcutaneous immunization by the topical application of a TLR agonist together with a CTL epitope might be an attractive way to deliver antigens for the treatment of malignancies or persistent virus infections. Directly targeting skin-resident APCs, TCI elicits a potent primary immune response [10]. As demonstrated by us previously, TCI-induced specific $\mathrm{T}$ cells rapidly fade away resulting in poor memory formation and insufficient protection in therapeutic tumor models $[6,13,14]$. The strength of the T-cell response can be enhanced by additional treatments like CD40 ligation or UV-B irradiation leading to memory formation $[12,13]$ and providing improved vaccination efficacy. Analysing the underlying mechanisms of TCI, we have revealed a suppressive effect of Treg cells, diminishing the induced CTL response [2]. Therefore, a promising advancement could be the combination of TCI with the inhibition of Treg cells. In this context, cyclophosphamide, a chemotherapeutic agent which is used for the treatment of melanoma, lymphomas and solid tumors, could be of interest $[22,23,33]$. Concerning its mode of action, $\mathrm{Cy}$ is described to decrease Treg cell numbers as well as to inhibit their suppressive capacity [29].

Combining low-dose Cy with TCI induced immune responses comparable to TCI alone, whereas higher doses suppressed the vaccination-induced CTL responses, most likely by its well-known lymphotoxic effects.

Working with low-dose Cy therefore seemed to be feasible. Towards the impact of $\mathrm{Cy}$ administered systemically on skin-resident DC targeted by TCI, we found that numbers of cells migrated from the skin to the draining lymph nodes are not affected (data not shown). Characterizing the phenotype of migrated DC revealed a more activated pattern, shown in upregulation of CD80 as well as CD86 after Cy-TCI compared to TCI alone. As bone-marrow derived $\mathrm{CD} 11 \mathrm{c}^{+}$dermal DCs are crucial in skin-associated immunity [12,34,35], an enhanced activated phenotype of skin-derived DC should support the induction of CTL responses which was not the case. Therefore, we suggest that beside Cy mediated enhancing effects, namely Treg cell depletion and DC activation, also inhibiting factors are induced, counteracting the stimulatory effects.

Recently Sevko and coworkers analysed low-dose Cy in a murine melanoma-model. Although this treatment results in reduced Treg cell levels and immunogenic tumor cell death upon Cy treatment, this treatment fails to increase mouse survival [31]. They show that the ineffective anti-tumor immunity is due to the production of various inflammatory mediators, such as GM-CSF, IL-1 $\beta$, IL-5, IL-10 IFN $\gamma$ and TNF- $\alpha$. Importantly, this leads to the expansion and activation of immunoregulatory myeloid-derived suppressor cells (MDSC) that accumulate especially in melanoma lesions. Transferring these findings to our setting, the 
induction of immunosuppressive immature myeloid-derived cells could counter-regulate the beneficial effects of Cy and TCI. Indeed, we found increased numbers of monocytic as well as granulocytic immature myeloid cells in the spleens of Cy-TCI treated mice. In addition, utilizing splenocytes from those mice as APCs in co-culture experiments after depleting either $\mathrm{CD} 25^{+}$or Ly6G/C $\mathrm{C}^{+}$cells revealed a strongly increased proliferation of responder $\mathrm{T}$ cells, leading to the conclusion that both cell types suppress CTL responses upon Cy treatment.

\section{Conclusions}

Taken together, we show that $\mathrm{Cy}$ does not diminish TCI-induced CTL-responses. Besides the disadvantage of immature myeloid cell formation and other suppressive influences, low-dose Cy mediates Treg cell reduction and DC activation, both desirable factors for vaccination. Therefore future vaccination protocols on the basis of $\mathrm{Cy}$ application may incorporate means to inhibit or modify immunosuppressive factors, e.g. accumulation of immature myeloid cells, to develop new therapeutic options against tumors by combining chemotherapy with immunomodulatory treatments.

\section{Acknowledgements}

This work was supported by $\mathrm{CI} 3$ Cluster (BMBF 131A035A), the GRK 1043 International Graduate School of Immunotherapy (HS) and "Forschungszentrum Immunologie (FZI)" of the University Medical Center Mainz.

The authors thank Andrea Drescher for excellent technical assistance.

Performed the experiments: JU, MW and PS. Analysed the data: JU, MW, PS and MPR. Contributed reagents/materials/analysis tools: HS. Wrote the paper: PS and MPR.

This work is part of the M.D. thesis of JU.

\section{REFERENCES}

[1] Navi D, Huntley A: Imiquimod 5 percent cream and the treatment of cutaneous malignancy. Dermatol Online J 2004 Feb 2;10:4.

[2] Stein P, Weber M, Prüfer S, Schmid B, Schmitt E, Probst HC, et al.: Regulatory $\mathrm{T}$ Cells and IL-10 Independently Counterregulate Cytotoxic T Lymphocyte Responses Induced by Transcutaneous Immunization. PLoS ONE 2011 Nov 16;6:e27911.

[3] Pardoll DM: The blockade of immune checkpoints in cancer immunotherapy. Nat Rev Cancer 2012 Apr;12:252-264.

[4] Romani N, Koide S, Crowley M, Witmer-Pack M,
Livingstone AM, Fathman CG, et al.: Presentation of exogenous protein antigens by dendritic cells to $\mathrm{T}$ cell clones. Intact protein is presented best by immature, epidermal Langerhans cells. J Exp Med 1989 Mar 1;169:1169-1178.

[5] Hemmi H, Kaisho T, Takeuchi O, Sato S, Sanjo H, Hoshino K, et al.: Small anti-viral compounds activate immune cells via the TLR7 MyD88-dependent signaling pathway. Nat Immunol 2002 Feb;3:196-200.

[6] Stoitzner P, Stoitzner P, Green LK, Green LK, Jung JY, Jung JY, et al.: Tumor immunotherapy by epicutaneous immunization requires langerhans cells. J Immunol $2008 \mathrm{Feb}$ 1;180:1991-1998.

[7] Hilleman MR: Vaccines in historic evolution and perspective: a narrative of vaccine discoveries. Vaccine $2000 \mathrm{Feb}$ $14 ; 18: 1436-1447$.

[8] Palamara F, Meindl S, Holcmann M, Lührs P, Stingl G, Sibilia M: Identification and characterization of pDC-like cells in normal mouse skin and melanomas treated with imiquimod. J Immunol 2004 Sep 1;173:3051-3061.

[9] Thomsen LL, Topley P, Daly MG, Brett SJ, Tite JP: Imiquimod and resiquimod in a mouse model: adjuvants for DNA vaccination by particle-mediated immunotherapeutic delivery. Vaccine 2004 Apr 16;22:1799-1809.

[10] Rechtsteiner G, Warger T, Osterloh P, Schild H, Radsak MP: Cutting edge: priming of CTL by transcutaneous peptide immunization with imiquimod. J Immunol 2005 Mar $1 ; 174: 2476-2480$.

[11] Sharma P, Wagner K, Wolchok JD, Allison JP: Novel cancer immunotherapy agents with survival benefit: recent successes and next steps. Nat Rev Cancer 2011 Nov;11:805-812.

[12] Stein P, Rechtsteiner G, Warger T, Bopp T, Fuhr T, Prüfer S, et al.: UV exposure boosts transcutaneous immunization and improves tumor immunity: cytotoxic T-cell priming through the skin. J Invest Dermatol 2011 Jan;131:211-219.

[13] Warger T, Warger T, Rechtsteiner G, Rechtsteiner G, Schmid B, Schmid B, et al.: Transcutaneous immunization with imiquimod is amplified by CD40 ligation and results in sustained cytotoxic T-lymphocyte activation and tumor protection. Clinic Rev Allerg Immunol 2007 Feb;32:57-66.

[14] Tsuyoshi Itoh EC: Transcutaneous Immunization with Cytotoxic T-Cell Peptide Epitopes Provides Effective Antitumor Immunity in Mice. J Immunother 2005;28:430.

[15] Kantoff PW, Higano CS, Shore ND, Berger ER, Small EJ, Penson DF, et al.: Sipuleucel-T Immunotherapy for Castration-Resistant Prostate Cancer 2010 Jul 29;363:411422.

[16] Walter S, Weinschenk T, Stenzl A, Zdrojowy R, Pluzanska A, Szczylik C, et al.: Multipeptide immune response to cancer vaccine IMA901 after single-dose cyclophosphamide associates with longer patient survival. Nat Med 2012 Jul 29; DOI: $10.1038 / \mathrm{nm} .2883$

[17] Tang Q, Bluestone JA: The Foxp3+ regulatory T cell: a jack of all trades, master of regulation. Nat Immunol 2008 Mar;9:239-244.

[18] Simpson TR, Li F, Montalvo-Ortiz W, Sepulveda MA, Bergerhoff K, Arce F, et al.: Fc-dependent depletion of tumor-infiltrating regulatory $\mathrm{T}$ cells co-defines the efficacy of 
anti-CTLA-4 therapy against melanoma. Journal of Experimental Medicine 2013 Aug 26;210:1695-1710.

[19] Sistigu A, Viaud S, Chaput N, Bracci L, Proietti E, Zitvogel L: Immunomodulatory effects of cyclophosphamide and implementations for vaccine design. Semin Immunopathol 2011 Jul;33:369-383.

[20] Aschan J, Carlens S, Hägglund H, Klaesson S, Mattsson J, Remberger M: Improved survival after bone marrow transplantation for early leukemia using busulfan-cyclophosphamide and individualized prophylaxis against graft-versus-host disease: a long-term follow-up. Clin Transplant 1999 Dec;13:512-519.

[21] Uchida S, Suzuki K, Akiyama S, Miyamoto M, Juji T, Fujiwara M: Suppressive effect of cyclophosphamide on the progression of lethal graft-versus-host disease in mice--a therapeutic model of fatal post-transfusion GVHD. Ther Immunol 1994 Dec;1:313-318.

[22] Koike N, Pilon-Thomas S, Mulé JJ: Nonmyeloablative chemotherapy followed by T-cell adoptive transfer and dendritic cell-based vaccination results in rejection of established melanoma. Journal of Immunotherapy 2008 May;31:402-412.

[23] Quan WDY, Quan FM, King LA, Walker PR: Low-dose cyclophosphamide and continuous-infusion interleukin-2 with famotidine in previously treated metastatic melanoma or kidney cancer. Cancer Biother Radiopharm 2008 Feb;23:108-113.

[24] Källén B, Dohlsten M, Klementsson H: Effect of cyclophosphamide pretreatment on autoimmune encephalomyelitis in rats. Acta Neurol Scand 1986 Apr;73:338-344.

[25] Polak L, Geleick H, Turk JL: Reversal by cyclophosphamide of tolerance in contact sensitization. Tolerance induced by prior feeding with DNCB. Immunology 1975 May;28:939942.

[26] Röllinghoff M, Starzinski-Powitz A, Pfizenmaier K, Wagner $\mathrm{H}$ : Cyclophosphamide-sensitive T lymphocytes suppress the in vivo generation of antigen-specific cytotoxic $\mathrm{T}$ lymphocytes. J Exp Med 1977 Feb 1;145:455-459.
[27] Yoshida S, Nomoto K, Himeno K, Takeya K: Immune response to syngeneic or autologous testicular cells in mice. I. Augmented delayed footpad reaction in cyclophosphamide-treated mice. Clin Exp Immunol 1979 Nov;38:211-217.

[28] Salem ML, Díaz-Montero CM, Al-Khami AA, El-Naggar SA, Naga O, Montero AJ, et al.: Recovery from cyclophosphamide-induced lymphopenia results in expansion of immature dendritic cells which can mediate enhanced prime-boost vaccination antitumor responses in vivo when stimulated with the TLR3 agonist poly(I:C). The Journal of Immunology 2009 Feb 15;182:2030-2040.

[29] Lutsiak MEC: Inhibition of $\mathrm{CD} 4+25+\mathrm{T}$ regulatory cell function implicated in enhanced immune response by low-dose cyclophosphamide. Blood 2005 Apr 1;105:28622868 .

[30] Lahl K, Loddenkemper C, Drouin C, Freyer J, Arnason J, Eberl G, et al.: Selective depletion of Foxp3+ regulatory $\mathrm{T}$ cells induces a scurfy-like disease. J Exp Med 2007 Jan 22;204:57-63.

[31] Sevko A, Sade-Feldman M, Kanterman J, Michels T, Falk CS, Umansky L, et al.: Cyclophosphamide promotes chronic inflammation-dependent immunosuppression and prevents antitumor response in melanoma. J Invest Dermatol 2013 Jun;133:1610-1619.

[32] Gabrilovich DI, Ostrand-Rosenberg S, Bronte V: Coordinated regulation of myeloid cells by tumours. Nat Rev Immunol 2012 Apr;12:253-268.

[33] Mitchell MS: Combinations of anticancer drugs and immunotherapy. Cancer Immunol Immunother 2003 Nov;52:686-692.

[34] Jung S, Unutmaz D, Wong P, Sano G-I, De los Santos K, Sparwasser T, et al.: In vivo depletion of $\mathrm{CD} 11 \mathrm{c}(+)$ dendritic cells abrogates priming of $\mathrm{CD} 8(+) \mathrm{T}$ cells by exogenous cell-associated antigens. Immunity 2002 Aug 1;17:211-220.

[35] Kissenpfennig A, Henri S, Dubois B, Laplace-Builhé C, Perrin P, Romani N, et al.: Dynamics and function of Langerhans cells in vivo: dermal dendritic cells colonize lymph node areas distinct from slower migrating Langerhans cells. Immunity 2005 May;22:643-654. 